\title{
Design and Realization of a Fuzzy Comprehensive Evaluation System for Music Teaching in Higher Education
}

\author{
https://doi.org/10.3991/ijet.v16i22.26875 \\ Zhiying Wang ${ }^{(\bowtie)}$, Dong Lyu \\ Hebei Institute of Communications, Shijiazhuang, China \\ ying $981677 @ 163$.com
}

\begin{abstract}
In recent years, music teaching in higher education has developed quickly, and attracted more and more attention. However, there is not yet a mature method to evaluate the education and teaching (ET) effect of music teaching in colleges. To solve the problem, this paper combines modern information technology and fuzzy comprehensive evaluation (FCE) to design and realize a FCE system for assessing the ET effect of music teaching in higher education. Firstly, the functional and non-functional requirements of the system were analyzed in detail. On this basis, the overall architecture and functional modules of the system were designed and realized. Application results show that the proposed system operated stably, and met the needs of ET effect evaluation. The research results are of theoretical and practical significance to the promotion of information-based teaching evaluation, the enhancement of management efficiency and quality, and the improvement of ET effect of music teaching in colleges.
\end{abstract}

Keywords - music, education and teaching (ET) effect, fuzzy comprehensive evaluation (FCE), design and realization

\section{$1 \quad$ Introduction}

The music teaching in higher education is very important for cultivating students' sentiments and improving their artistic literacy and aesthetic ability. In China, the music teaching in higher education started late, but it develops very fast in recent years and receives more and more attention from people, however, survey reveals that the various links of music teaching in higher education are incomplete and immature [1]. ET effect evaluation is a key link and a hard work, and developing a sound ET effect evaluation system can effectively solve this problem [2], so how to develop a scientific, reasonable, and adaptable ET effect evaluation system is an issue that should be addressed and solved for improving the teaching quality and management work efficiency of music class in higher education.

With the penetration of information technology in modern education, informatized teaching is now an inevitable trend in the field, and many field scholars and experts have conducted all kinds of research on how to apply information technology to assist 
classroom teaching and improve its quality and they have achieved remarkable results [3]. In some foreign countries, the research on ET effect evaluation started earlier, and a few of them have built mature and complete ET effect evaluation systems, such as British, America, and Japan have formulated unified and standardized requirements for teaching quality management [4]. In China, existing works concerning ET quality and effect evaluation are mostly theoretical discussions, most of the field scholars focused on aspects such as the content and structure of ET effect evaluation indicators, and they built various evaluation systems [5]. Principal component analysis (PCA), analytic hierarchy process (AHP), BP neural network, and fuzzy comprehensive evaluation (FCE) are common methods adopted in ET effect evaluation in the Chinese academic circle [6-12]. Among the four, FCE is a comprehensive evaluation method established based on fuzzy mathematics, it can effectively solve fuzzy decision-making questions with multiple factors that are hard to quantify; since it can convert qualitative evaluation into quantitative evaluation, the method is very suitable for solving various non-deterministic problems [13]. Due to these merits, FCE has been widely used in ET effect evaluation studies, however, after carefully reviewing relevant literatures, it's found that few researches have applied it to studying the ET effect evaluation of music teaching in higher education in China [14].

Out of these concerns, this paper took the ET effect evaluation of music teaching in higher education as the entry point, drawing on the FCE method, this paper designs and develops a FCE system for assessing the ET effect of music teaching in higher education, elaborates the design of the FCE-based ET effect evaluation model of music teaching in higher education; then, the paper analyzes the functional requirements of the system, and determines the overall architecture of the ET effect evaluation system and its function modules. The proposed system and model could contribute to improving the teaching quality and management efficiency of music teaching in higher education.

\section{$2 \quad$ Modeling}

\subsection{EIS proposal}

The ET effect evaluation of music teaching in higher education is to evaluate each kind of objects that appear during the teaching process, and it is an important means to propel students to grow and develop, and to improve the teaching quality of teachers [15]. Referring to relevant teaching effect evaluation studies, existing teaching quality evaluation indicator systems, and the characteristics of music teaching in higher education, an FCE-based EIS was established for the music teaching in higher education, as shown in Table 1 [16]. 
Table 1. The proposed FCE-based EIS

\begin{tabular}{|c|c|c|c|c|}
\hline $\begin{array}{l}\text { Evaluation } \\
\text { index }\end{array}$ & First-level index & Weight & Second-level index & Weight \\
\hline \multirow{15}{*}{$\begin{array}{l}\text { Teaching ef- } \\
\text { fect } X\end{array}$} & \multirow{2}{*}{ Teaching objectives $\mathrm{X} 1$} & \multirow{2}{*}{ w1 } & $\begin{array}{c}\text { Reflect the requirements of the syllabus and } \\
\text { the characteristics of music teaching X11 }\end{array}$ & w11 \\
\hline & & & $\begin{array}{l}\text { Clear goals, throughout, in line with the } \\
\text { cognitive law of college students X12 }\end{array}$ & w12 \\
\hline & \multirow{3}{*}{ Teaching content $\mathrm{X} 2$} & \multirow{3}{*}{ w2 } & $\begin{array}{l}\text { Teaching content meets the requirements of } \\
\text { teaching objectives X21 }\end{array}$ & w21 \\
\hline & & & $\begin{array}{c}\text { The content arrangement is scientific and } \\
\text { reasonable, close to the age of the students, } \\
\text { and combines theory and practice X } 22\end{array}$ & w22 \\
\hline & & & $\begin{array}{c}\text { The key points and difficulties of teaching } \\
\text { are handled properly X23 }\end{array}$ & w22 \\
\hline & \multirow{3}{*}{ Teaching method X3 } & \multirow{3}{*}{ w3 } & Flexible and targeted X31 & w31 \\
\hline & & & \begin{tabular}{|c|} 
Teach students in accordance with their ap- \\
titude, focus on inspiration and inducement, \\
have individuality and creativity X 32
\end{tabular} & w32 \\
\hline & & & \begin{tabular}{|c|} 
Reasonable use of auxiliary teaching facili- \\
ties (such as musical instruments, multime- \\
dia, etc.) to stimulate students' interest in \\
learning X33
\end{tabular} & w33 \\
\hline & \multirow{3}{*}{ Teaching skills X4 } & \multirow{3}{*}{ w4 } & $\begin{array}{l}\text { Whether the music learning demonstration } \\
\text { is proficient, natural, and contagious X41 }\end{array}$ & w41 \\
\hline & & & Clear language, fluent and expressive X42 & w42 \\
\hline & & & $\begin{array}{c}\text { Natural teaching attitude, dignified appear- } \\
\text { ance X43 } \\
\end{array}$ & w43 \\
\hline & \multirow{4}{*}{ Teaching effect X5 } & \multirow{4}{*}{ w5 } & $\begin{array}{l}\text { Achieve the expected teaching goals and } \\
\text { the teaching quality is good X51 }\end{array}$ & w51 \\
\hline & & & $\begin{array}{l}\text { Pay attention to the cultivation of students' } \\
\text { music aesthetic ability, creative ability and } \\
\text { thinking ability X52 }\end{array}$ & w52 \\
\hline & & & $\begin{array}{l}\text { Students can better master music } \\
\text { knowledge and skills X53 }\end{array}$ & w53 \\
\hline & & & $\begin{array}{l}\text { The classroom atmosphere is good, teach- } \\
\text { ers and students interact with each other } \\
\text { X54 }\end{array}$ & w54 \\
\hline
\end{tabular}

\subsection{Index weight value determination}

Since the ET effect evaluation data sets contain large data volume and the score of ET effect is a discrete variable, in order to avoid the influence of subjective factors and improve the efficiency and accuracy of data separation, this paper adopted the K-means algorithm in machine learning as the classification algorithm; before applying it, the Kmeans algorithm had been optimized to overcome the traditional K-means algorithm's inability to solve the problem of the discrete vector of ET effect score that is distributed in $0-1[17]$. 
Application of the optimized K-means algorithm in the ET effect evaluation system. First, the data sets were processed; a single evaluation result can be expressed as:

$$
\dot{X_{k}}=\left(X_{11}, X_{12}, X_{21} \cdots X_{54}\right)
$$

where, $\overrightarrow{X k}$ represents the $\mathrm{k}$-th evaluation result in the evaluation process.

Multiple evaluation results were combined to get the dataset matrix $\mathrm{X}$ as:

$$
X=\left|\begin{array}{c}
X_{1} \\
\cdots \\
\overrightarrow{X_{n}}
\end{array}\right|=\left[\begin{array}{ccc}
X_{111} & \cdots & X_{154} \\
\cdots & \ddots & \cdots \\
X_{n 11} & \cdots & X_{n 54}
\end{array}\right]
$$

Second, four vectors $\overrightarrow{n D}, \overrightarrow{n E}, \overrightarrow{n F}$, and $\overrightarrow{n G}$ were used to represent four random vectors that are relatively close to the focus point $(1,1, \ldots 1)$.

Third, Formula 3 was used to calculate the Euclidean distance from each single vector to $\overrightarrow{n D}, \overrightarrow{n E}, \overrightarrow{n F}$, and $\overrightarrow{n G}$, and the minimum value was taken; then, each data point in set $X$ was assigned to the cluster closest to it, namely the four grades of excellent(D), $\operatorname{good}(\mathrm{E}), \operatorname{medium}(\mathrm{F})$, and qualified(G) [18].

$$
d\left(\overrightarrow{x_{i}}, \overrightarrow{n_{i}}\right)=\left\|\overrightarrow{x_{i}}-\overrightarrow{n_{i}}\right\|
$$

Table 2 shows the evaluation score intervals and the four corresponding grades.

Table 2. Evaluation score intervals and grades

\begin{tabular}{|l|c|}
\hline \multicolumn{1}{|c|}{ Score } & Grade \\
\hline $0.9-1$ & Excellent \\
\hline $0.8-0.9$ & Good \\
\hline $0.6-0.8$ & Medium \\
\hline $0.1-0.6$ & Qualified \\
\hline
\end{tabular}

According to formulas (4)-(6), vectors $\overrightarrow{n D}, \overrightarrow{n E}, \overrightarrow{n F}$, and $\overrightarrow{n G}$ updated until the cluster identification vector $\vec{n}$ no longer changed [19].

$$
\begin{gathered}
\overrightarrow{n_{D}}=\frac{1}{\operatorname{crad}(D)}\left(\sum_{x i \in D} \vec{x}_{i}\right) \\
\overrightarrow{n_{E}}=\frac{1}{\operatorname{crad}(D)+\operatorname{crad}(E)}\left(\sum_{x i \in D} \overrightarrow{x_{i}}+\sum_{x i \in E} \overrightarrow{x_{j}}\right) \\
\overrightarrow{n_{F}}=\frac{1}{\operatorname{crad}(D)+\operatorname{crad}(E)+\operatorname{crad}(F)}\left(\sum_{x i \in D} \overrightarrow{x_{i}}+\sum_{\vec{x} i \in E} \overrightarrow{x_{j}}+\sum_{\vec{x} i \in F} \overrightarrow{x_{k}}\right)
\end{gathered}
$$




$$
\overrightarrow{n_{G}}=\frac{1}{\operatorname{crad}(D)+\operatorname{crad}(E)+\operatorname{crad}(F)+\operatorname{crad}(G)}\left(\sum_{\overrightarrow{x i \in D}} \overrightarrow{x_{i}}+\sum_{\overrightarrow{x i} \in E} \overrightarrow{x_{j}}+\sum_{\overrightarrow{x i} \in F} \overrightarrow{x_{k}}+\sum_{\vec{x} i \in G} \overrightarrow{x_{l}}\right)
$$

where, the numbers of vectors in vector sets D, E, F, and G were represented by $\operatorname{crad}(D), \operatorname{crad}(E), \operatorname{crad}(F)$, and $\operatorname{crad}(G)$, respectively.

Weight value determination. Formula $(8)$ was used to determine the weight values of indexes at all levels [20].

$$
p\left(J \mid a_{i}\right)=\frac{1}{\sqrt{2 \pi} \sigma^{2}} \exp \left(\frac{a_{i}-\mu}{2 \sigma^{2}}\right)
$$

where, $\mu$ and $\lambda$ were respectively calculated from the mean and variance of the samples.

Evaluation of ET effect. According to the above text, the weight values of indexes at all levels could be calculated:

$$
\begin{gathered}
\vec{X}=\left(w_{1}, w_{2}, w_{3}, w_{4}, w_{5}\right) \\
\overrightarrow{X_{1}}=\left(w_{11}, w_{12}\right) \\
\overrightarrow{X_{2}}=\left(w_{21}, w_{22}, w_{23}\right) \\
\overrightarrow{X_{3}}=\left(w_{31}, w_{32}, w_{33}\right) \\
\overrightarrow{X_{4}}=\left(w_{41}, w_{42}, w_{43}\right) \\
\overrightarrow{X_{5}}=\left(w_{51}, w_{52}, w_{53}, w_{54}\right)
\end{gathered}
$$

The teacher evaluation result was assumed to be:

$$
\overrightarrow{r_{i}}=\left(t_{1}, t_{2}, t_{3}, t_{4}\right)
$$

$t_{1}, t_{2}, t_{3}, t_{4}$ represented the proportions of the four grades (excellent, good, medium, qualified), $\vec{r} \vec{\imath}$ represented the $i$-th second-level index.

$R_{i}$ represented the evaluation matrix of first-level evaluation index $X_{i}(i=1,2,3,4,5)$.

$$
R_{i}=\left[\begin{array}{c}
\overrightarrow{r_{1}} \\
\vdots \\
\overrightarrow{r_{n}}
\end{array}\right]
$$

The data was normalized, after calculation, the weight vector of $X_{i}$ was obtained as:

$$
\overrightarrow{w_{i}}=X_{i} \times R_{i}=\left(b_{1}, b_{2}, b_{3}, b_{4}\right)
$$


where, $b 1, b 2, b 3, b 4$ represented the probability values of first-level indexes belonging to the four grades (excellent, good, medium, qualified).

In the same way, the weight vectors $\overrightarrow{w 2}, \overrightarrow{w 3}, \overrightarrow{w 4}, \overrightarrow{w 5}$ of $X_{2}, X_{3}, X_{4}, X_{5}$ were calculated as well.

Above steps were repeated until the weight vecotr of the comprehensive evaluation index $X$ was obtianed:

$$
\vec{S}=\vec{X}\left[\begin{array}{l}
\overrightarrow{w_{1}} \\
\overrightarrow{w_{2}} \\
\overrightarrow{w_{3}} \\
\overrightarrow{w_{4}} \\
\overrightarrow{w_{5}}
\end{array}\right]=\left(S_{1}, S_{2}, S_{3}, S_{4}\right)
$$

The principle of the maximum degree of membership was taken as the final evaluation criterion:

$$
\text { Score }=\max _{s i \in S}\left\{s_{i}\right\}
$$

Through the above calculation process of the proposed FCE model, the weight values of the corresponding evaluation indexes, and the final evaluation results of the objects could be obtained.

\section{Design and realization of the FCE system for music teaching in higher education}

\subsection{System requirement analysis}

Functional requirements. The proposed system was developed based on the idea of function modularization. According to the survey and research results of engaged teachers and teaching work administrators, this paper designed a FCE system for assessing the ET effect of music teaching in higher education, and the system is consisted of five main modules, as shown in Figure 1 [21]. 


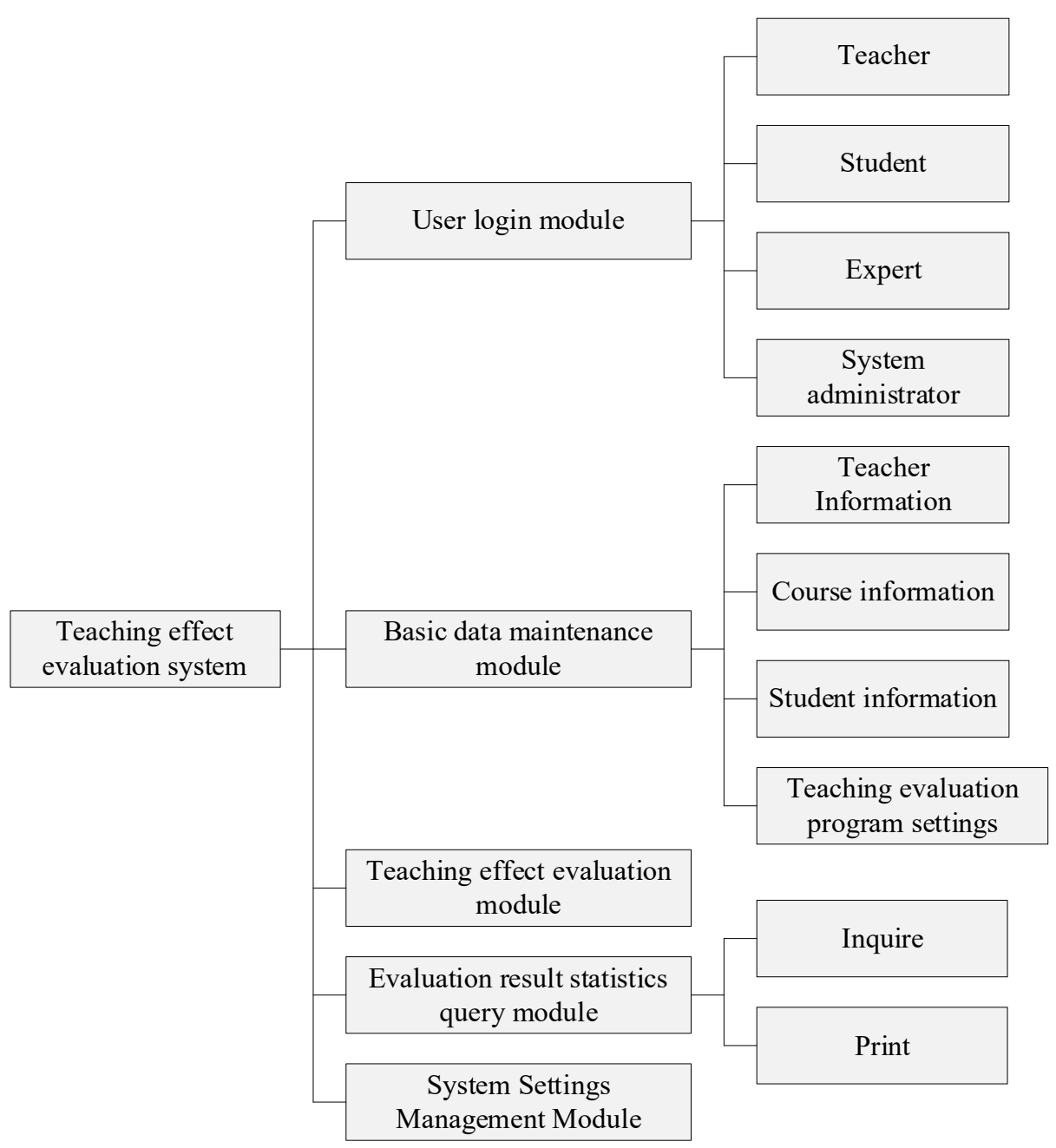

Fig. 1. Function modules of the proposed system

User login module. The proposed evaluation system involves three parts: student evaluation, teacher self-evaluation, and expert evaluation. To ensure normal and stable operation, the system needs maintenance and management, therefore, besides student users, teacher users, and expert users, the system also needs administrator users. To ensure system safety and prevent private data leakage, all types of users are required to enter the correct username and password and they could only access to corresponding authorized interfaces after passing system verification, then they could perform various operations such as changing the personal profile or password, giving ET effect evaluation, and managing and maintaining the system, etc. [22]. 
Basic data maintenance module. Since teachers' teaching tasks and lecturing classes are different in each semester, before giving ET effect evaluation, the system administrators and teachers should update basic data such as the information of teachers, students, and courses. Besides, the EIS isn't static, but should be modified regularly according to the actual teaching situations, such as adding or deleting evaluation indexes, or altering the content of evaluation indexes, or inputting the weight values of evaluation indexes, etc. [23].

Teaching effect evaluation module. This module is the core function module in the proposed system, after logging in to the system, students, teachers, and experts could enter this module and give online evaluation to the ET effect of teachers according to the evaluation indexes that had been already input in the system; after finishing the evaluation, the evaluation results are submitted to the system, and a database of ET effect evaluation could be formed; then, the system will assess the ET effect of teachers according to the designed FCE model and give the final quantified evaluation grades [24].

Evaluation result statistics and query module. After finishing ET effect evaluation, the system will automatically count and analyze the teaching effect evaluation of teachers, teachers, experts, and school management staffs who have participated in the evaluation could query the ET effect evaluation results and history evaluation records. The system also provides users with the function to query the evaluation results under different statistical conditions, such as you can query the evaluation results of a teacher in different years, different classes, or in different courses, etc., in this way, comparison of the teacher's performance could be carried out horizontally and/or vertically; moreover, the system can also print out the evaluation results.

System configuration and management module. System administrators are the main users of the system configuration and management module. They can use this module to manage the users, user rights, and system log information, etc., so as to ensure safe and stable system operation and satisfy functional requirements of different users. Of course, other users can also make simple settings using this module, but it's only limited to the setting of their personal information and personalized interface.

Non-functional requirements. In addition to ensuring safe and stable operation of system functions, the system should have well-designed operation interfaces, the operation process shouldn't be too complicated, users should be able to operate the system easily; moreover, the system's maximum number of concurrent users should be greater than 200 to ensure that multiple users can log in to the system at the same time to give ET effect evaluation. At last, the system should also have a good compatibility to connect with other campus systems (such as student profile management system, or the educational administration system, etc.).

\subsection{Overall architecture of the proposed system}

To facilitate users to give ET effect evaluation anywhere, anytime, on campus, or off campus, the proposed system adopted a three-layer BS structure, as shown in Figure $2[25]$. 


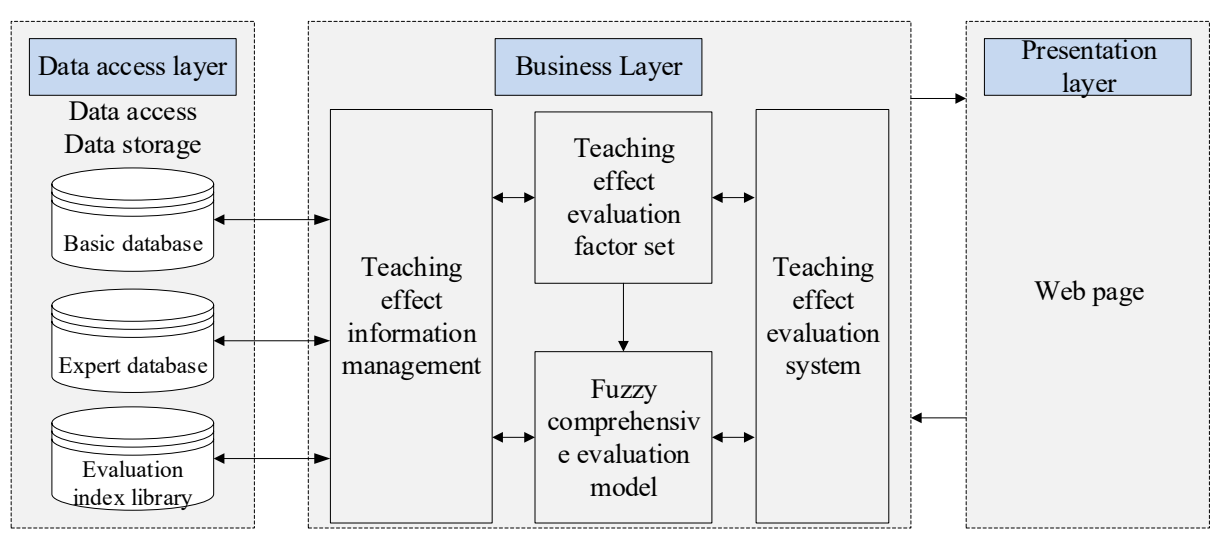

Fig. 2. Overall architecture of the proposed system

\subsection{Design and implementation of system function modules}

Due to limited space, this paper took the student evaluation and evaluation result statistics and analysis functions in the core module, the teaching effect evaluation module as an example to introduce the design of the module and its realization.

Student evaluation. A student user enters the right username and password and selects the corresponding user type, then he/she enters the personal main page, selects the teaching effect evaluation module, and gives evaluation on teachers who need to be evaluated, after completing the evaluation, the results are submitted, and the system automatically judges the data validity of the submitted results, if there's missing content, the system will alert and ask the student to answer again, if the validity check is past, the results are submitted successively. If there's an error in the username or password, the system will pop up a prompt to tell the user that he/she cannot enter the system. Figure 3 shows the activity flow of the student teaching effect evaluation module. Figure 4 shows the system login interface. Figure 5 shows the student evaluation interface. 
Paper-Design and Realization of a Fuzzy Comprehensive Evaluation System for Music Teaching in...

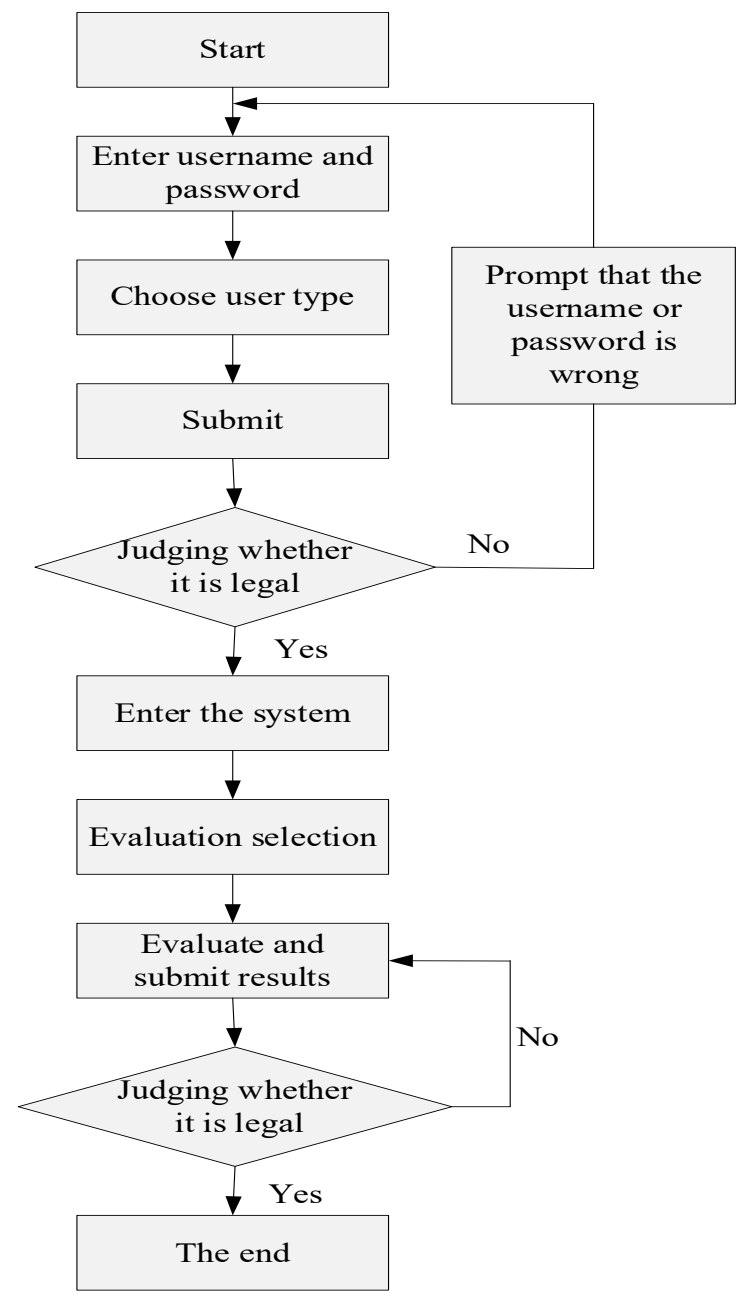

Fig. 3. Activity flow of the student teaching effect evaluation module

\section{Teaching effect evaluation system}

Username:

Password:

User type:

Log in Register

Fig. 4. System login interface 


\begin{tabular}{|c|c|c|}
\hline The fourth $\mathrm{pa}$ & Teaching skills & \\
\hline 9. Whether th & e music learning demonst & tion is proficient, natural and contagious \\
\hline oExcellent & $\circ$ Good $\quad$ Medium & ०Qualified \\
\hline 10.Clear lang & uage, fluent and expressi & \\
\hline oExcellent & $\circ$ Good $\quad$ Medium & ०Qualified \\
\hline 11.Natural te & aching attitude, dignified & opearance \\
\hline oExcellent & $\circ$ Good oMedium & ०Qualified \\
\hline
\end{tabular}

Fig. 5. Student evaluation interface

Statistics and analysis of evaluation results. After all student evaluations, expert evaluations and teacher self-evaluations are completed, the system will store relevant data and analyze the teaching effect results of the teachers. Administrators, teachers, and experts can log in to the system to query the evaluation results, and they can compare and analyze the results of multiple teachers. After selecting evaluation conditions, users can get the corresponding evaluation interface. Figure 6 shows the basic flow of the statistics and analysis of the evaluation results.

Figure 7 shows the statistics and analysis interface of the evaluation results of different teachers who teach a same course, if administrators or experts want to check the specific differences among teachers, they can click to view the scores of each item of the teachers.

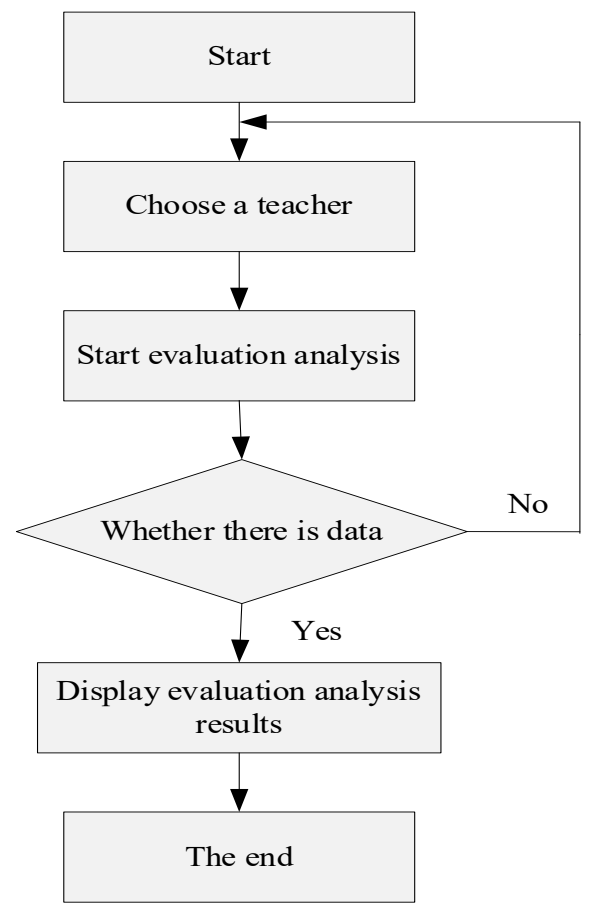

Fig. 6. Basic flow of statistics and analysis of evaluation results 
Paper-Design and Realization of a Fuzzy Comprehensive Evaluation System for Music Teaching in...

\begin{tabular}{c|c|c|c|c|c|c}
$\begin{array}{c}\text { Serial } \\
\text { number }\end{array}$ & Name & Job title & Teaching Courses & Rating & Score & $\begin{array}{c}\text { Evaluation } \\
\text { Analysis }\end{array}$ \\
\hline 1 & Liu Qiang & Lecturet & Music Appreciation & Good & 0.83 & $\underline{\text { View }}$ \\
\hline 2 & Fu Huan & Professor & Music Appreciation & Excellent & 0.92 & $\underline{\text { View }}$ \\
\hline 3 & Li Yiyi & $\begin{array}{c}\text { Associate } \\
\text { Professor }\end{array}$ & Music Appreciation & Excellent & 0.95 & $\underline{\text { View }}$ \\
\hline 4 & Hou Yu & Teaching assistant & Music Appreciation & Medium & 0.78 & $\underline{\text { View }}$ \\
\hline 5 & Zhang Xin & Lecturet & Music Appreciation & Medium & 0.62 & $\underline{\text { View }}$
\end{tabular}

Fig. 7. Statistics and analysis interface of evaluation results of different teachers teaching a same course

\section{Conclusions}

Based on FCE theory, this paper designed and implemented a FCE system for assessing the ET effect of music teaching in higher education, and obtained following conclusions:

1. This paper constructed an EIS and a FCE model for assessing the ET effect of music teaching in higher education.

2. This paper analyzed the functional and non-functional requirements of the system, divided the system into five functional modules, and designed the overall architecture of the system.

3. Taking the student evaluation and evaluation result statistics and analysis functions in the core module, the teaching effect evaluation module as an example, this paper introduced the design of the module and its realization in detail, and the results showed that the system could operate normally and stably, and it could meet the requirements of the evaluation of the ET effect of music teaching in higher education.

\section{$5 \quad$ References}

[1] Bing, W., Wang, S.L., Li, L. (2010). Fuzzy comprehensive evaluation of district heating systems. Energy Policy, 38(10): 5947-5955. https://doi.org/10.1016/j.enpol.2010.05.048

[2] Yang, J., Shen, L.Q., Jin, X.Y., Hou, L.Y., Shang, S.M., Zhang, Y. (2019). Evaluating the quality of simulation teaching in fundamental nursing curriculum: AHP-fuzzy comprehensive evaluation. Nurse Education Today, 77: 77-82. https://doi.org/10.1016/ j.nedt.2019.03.012

[3] Huang, W., Li, N., Qiu, Z.J., Jiang, N., Wu, B., Liu, B. (2020). An automatic recognition method for students' classroom behaviors based on image processing. Traitement du Signal, 37(3): 503-509. https://doi.org/10.18280/ts.370318

[4] Zhang, C., Liu, F., Jing, W. (2010). Set pair mathematical model of teaching quality evaluation system and its application. IEEE, 531-535. https://doi.org/10.1109/hsi.2010 .5514516 
Paper-Design and Realization of a Fuzzy Comprehensive Evaluation System for Music Teaching in...

[5] Zhou, N., Zhang, Z.F., Li, J. (2020). Analysis on course scores of learners of online teaching platforms based on data mining. Ingénierie des Systèmes d'Information, 25(5): 609-617. https://doi.org/10.18280/isi.250508

[6] Killian, J.K.N. (1981). Effect of instructions and feedback on music teaching skills. Journal of Music Therapy, 18(4): 166-180. https://doi.org/10.1093/jmt/18.4.166

[7] Maji S., Kanrar S. (2019). SpliceCombo: A hybrid technique efficiently use for principal component analysis of splice site prediction, Ingenierie des Systemes d'Information, 24(1): 67-75. https://doi.org/10.18280/isi.240110

[8] Zhang, F., Zhang, C., Yang, H.M., Zhao, L. (2019). Point cloud denoising with principal component analysis and a novel bilateral filter. Traitement du Signal, 36(5): 93-398. https://doi.org/10.18280/ts.360503

[9] Wang, W.X. (2019). Site selection of fire stations in cities based on geographic information system (GIS) and fuzzy analytic hierarchy process (FAHP). Ingénierie des Systèmes d'Information, 24(6): 619-626. https://doi.org/10.18280/isi.240609

[10] Huang, Q., Cui, L.M. (2019). Design and application of face recognition algorithm based on improved backpropagation neural network. Revue d'Intelligence Artificielle, 33(1): 25-32. https://doi.org/10.18280/ria.330105

[11] Mohammedhasan, M., Uğuz, H. (2020). A new early stage diabetic retinopathy diagnosis model using deep convolutional neural networks and principal component analysis. Traitement du Signal, 37(5): 711-722. https://doi.org/10.18280/ts.370503

[12] Yang, Z.H. (2020). Analysis of the impacts of open residential communities on road traffic based on AHP and fuzzy theory. Ingénierie des Systèmes d'Information, 25(2): 183-190. https://doi.org/10.18280/isi.250205

[13] Zhang, C.Y., Liu, F.C., Wang, J. (2010). Set pair mathematical model of teaching quality evaluation system and its application. IEEE, 589-592. https://doi.org/10.1109/hsi.2010. 5514516

[14] Jylhä, A., Ekman, I., Erkut, C., Tahiroglu, K. (2011). Design and evaluation of humancomputer rhythmic interaction in a tutoring system. Computer Music Journal, 35(2): 36-48. https://doi.org/10.1162/comj_a 00055

[15] Feng, S., Xu, L.D. (1999). An intelligent decision support system for fuzzy comprehensive evaluation of urban development. Expert Systems with Applications, 16(1): 21-32. https://doi.org/10.1016/s0957-4174(98)00028-1

[16] Zandén, O., Thorgersen, C.F. (2015). Teaching for learning or teaching for documentation? music teachers' perspectives on a Swedish curriculum reform. British Journal of Music Education, 32(1): 37-50. https://doi.org/10.1017/s0265051714000266

[17] Lv, H.Z., Luo, J.Y. (2021). Creative approaches in music teaching: possibilities of web 2.0 technologies. Thinking Skills and Creativity, 40(1): 100840. https://doi.org/10.1016 j.tsc. 2021.100840

[18] Henkel, M. (1997). Teaching quality assessments: public accountability and academic autonomy in higher education. Evaluation, 3 (1): 9-23. https://doi.org/10.1177/135638909 700300102

[19] Li, W.J., Liang, W., Zhang L.B., Tang, Q. (2015). Performance assessment system of health, safety and environment based on experts' weights and fuzzy comprehensive evaluation. Journal of Loss Prevention in the Process Industries,35, 95-103. https://doi.org/10.1016/ j.jilp.2015.04.007

[20] Tang, L., Cao, Y., Liu, Z., Qian, K., Zhou, Y.S. (2020). Improving the quality of preclinical simulation training for dental students using a new digital real-time evaluation system. European Journal of Dental Education, 25(1): 100-107. https://doi.org/10.1111/eje.12580 
Paper-Design and Realization of a Fuzzy Comprehensive Evaluation System for Music Teaching in...

[21] Toby, S. (1993). Class size and teaching evaluation: or, the 'general chemistry effect' revisited. Journal of Chemical Education, 70 (6): 465-466. https://doi.org/10.1021/ ed070p465

[22] Mcowen, K.S., Kogan, J.R., Shea, J.A. (2008). Elapsed time between teaching and evaluation: does it matter. Academic Medicine, 83 (Supplement), 29-32. https://doi.org/ $10.1097 / \mathrm{acm} .0 \mathrm{~b} 013 \mathrm{e} 318183 \mathrm{e} 37 \mathrm{c}$

[23] Lu, J., Ma, J., Zhang, G.Q., Zhu, Y.J., Zeng, X.Y., Koehl, L. (2011). Theme-based comprehensive evaluation in new product development using fuzzy hierarchical criteria group decision-making method. IEEE Transactions on Industrial Electronics, 58(6): 22362246. https://doi.org/10.1109/tie.2010.2096171

[24] Ji, S., Li, X., Ma, Y., Cai, H. (2000). Optimal tolerance allocation based on fuzzy comprehensive evaluation and genetic algorithm. International Journal of Advanced Manufacturing Technology, 16(7): 461-468. https://doi.org/10.1007/s001700070053

[25] McOwen, K.S., Kogan, J.R., Shea, J.A. (2008). Elapsed time between teaching and evaluation: does it matter. Academic Medicine, 83(Supplement): 29-32. https://doi.org/ $\underline{10.1097 / \mathrm{acm} .0 \mathrm{~b} 013 \mathrm{e} 318183 \mathrm{e} 37 \mathrm{c}}$

\section{Authors}

Zhiying Wang, Lecturer, Master of Fine Arts, graduated from Hebei Normal University in 2012 and currently works at Hebei Institute of Communications. The main research areas are vocal singing and teaching, and musical education.

Dong Lyu, Lecturer, Master of Arts, graduated from Yunnan Art University in 2010 and currently works at Hebei Institute of Communications. The main research areas are music production and recording, and musical education (Email: winter886666@163.com).

Article submitted 2021-09-14. Resubmitted 2021-10-11. Final acceptance 2021-10-12. Final version published as submitted by the authors. 